\title{
Le ciblage des politiques agricoles en Inde: les implications pour les paysans du système d'approvisionnement public
}

\author{
Christine Lutringer* \\ Centre Albert Hirschman sur la démocratie, Institut des hautes études internationales et du développement, Genève, Suisse
}

\begin{abstract}
Résumé - Cette contribution examine un volet stratégique de l'intervention publique dans le secteur agricole de l'Inde. Mis en place dans les années 1960, le système d'achat public et de prix minimum de soutien joue depuis lors un rôle pivot dans la politique de sécurité alimentaire. Ce système vise à garantir une rémunération suffisante pour les paysans, la constitution de stocks et une distribution publique aux consommateurs les plus pauvres. Le gouvernement utilise également la politique de prix pour stimuler la production de denrées agricoles spécifiques car les prix de soutien influencent les décisions des paysans sur l'allocation des cultures et des types de production. Concrètement, il vise à atteindre des quantités minimales de grains alimentaires dans le pays. Cette politique a conduit au ciblage de certaines denrées, régions et formes de production. L'article analyse la logique du système d'achat et ses effets pour les paysans, à la fois économiques et sociopolitiques. Il montre que les débats actuels sur les réformes en cours portent sur un ciblage plus explicite des catégories de paysans et des régions que ce système pourrait servir à promouvoir.
\end{abstract}

Mots clés : politiques de prix agricoles / approvisionnement public / révolution verte / ciblage / Inde

\begin{abstract}
Targeting agricultural policies in India: some implications for farmers of the public procurement system. This contribution discusses a strategic aspect of the public intervention in India's agricultural sector. Created in the 1960 s, the system of public procurement and minimum support prices has played since then a key role in food security policies. It aims to provide basic earnings for farmers, to build buffer-stocks and to supply the public distribution system that is geared towards the poorest consumers. Minimum support prices influence farmers' decisions on the allocation of the different varieties and crops. The government therefore uses the pricing policy to stimulate the production of specific food grains. Concretely, it seeks to procure minimum quantities of food grain. This policy has led to target and favour specific crops, regions and forms of production. This paper examines the rationale of the system and its effects on farmers, both economic and socio-political. It also shows that ongoing reforms are leading to a more explicit targeting of the system towards selected categories of farmers and of regions that this system could promote.
\end{abstract}

Keywords: agricultural pricing policies / public procurement / green revolution / targeted policies / India

\section{Introduction}

L'Inde constitue un pays de référence pour l'agriculture mondiale. D'un point de vue international, le secteur agricole indien impressionne par sa taille et par l'importance de la production: employant plus de 250 millions de personnes, l'Inde figure en effet parmi les plus gros producteurs mondiaux de riz, coton, thé, canne à sucre, jute, poivre, lait ou œufs. Elle se positionne ainsi comme un acteur important

\footnotetext{
* Auteur de correspondance :

christine.lutringer@graduateinstitute.ch
}

dans le commerce agroalimentaire mondial (Pouch et Kheraief, 2016). Cependant, dès que l'on introduit le paramètre démographique et la question des disponibilités alimentaires par habitant, la sécurité alimentaire apparaît clairement comme un défi de taille. Le secteur agricole concentre un grand nombre d'enjeux de développement pour le pays. Sur le plan national, l'évolution de ce secteur est au centre de l'attention des pouvoirs publics: l'agriculture occupe encore aujourd'hui plus de la moitié de la population active, un chiffre quasi-stable depuis une dizaine d'années, alors que sa part dans le Produit intérieur brut (PIB), équivalent à 17,4\% en 2015-2016 (Government of India, 2016a), est en diminution lente mais constante. La croissance 
agricole est faible rapportée au taux de croissance économique du pays : le secteur agricole a enregistré un taux de croissance annuel moyen de 3,8\% depuis 2004-2005 et de seulement $1,1 \%$ en 2015-2016 (Government of India, 2016a). Par ailleurs, 69,4\% des exploitations agricoles s'étendent sur moins d'un hectare et sont considérées par les institutions publiques comme «marginales » (NSSO, 2014). De manière générale, le processus de fragmentation des exploitations agricoles indiennes et la diminution de leur taille moyenne pose la question de leur viabilité. On estime que les quatre cinquièmes des exploitations indiennes ne seraient pas viables sans revenu supplémentaire (Singh, 2012). Au demeurant, la croissance du secteur non agricole ne s'est pas traduite par une création suffisamment importante d'emplois ruraux, comme le montrent Dorin et Aubron (2016) qui analysent la trajectoire contemporaine du secteur agricole indien. D'où l'importance de l'intervention publique, tant dans le domaine agricole que pour stimuler les revenus non agricoles.

Dans ce contexte, les politiques publiques doivent contribuer à promouvoir un développement agricole à la fois plus efficace et plus équitable. Théoriquement, l'une des stratégies qui combine ces deux objectifs est celle du ciblage: selon Lavallée et al. (2009), le ciblage permet d'une part la mise en œuvre de «programmes élaborés spécifiquement pour répondre aux besoins des pauvres. D'autre part, en concentrant les ressources sur les pauvres, [il] permet une utilisation plus efficace des ressources qu'une politique universelle. » Cette stratégie est généralement opposée aux politiques dites universalistes. Or, dans le cas de la politique indienne d'intervention sur les marchés agricoles, c'est un entre-deux qui a été mis en place. Tout particulièrement en matière céréalière, le système d'achat de la production par l'État est au centre d'un dispositif de sécurité alimentaire. Il se situe au croisement des grandes priorités de la politique agricole, à la fois en matière de sécurité alimentaire et de développement agricole. De fait, l'État considère que certaines productions sont essentielles pour la sécurité alimentaire. Pour donner un ordre de grandeur, l'objectif pour l'approvisionnement public en blé est de 30,5 millions de tonnes pour l'année 2016 (Government of India, 2016b). Ce système d'achat public, de stockage et de distribution, probablement le plus grand au monde (Landy, 2006), est l'instrument central de la politique agricole indienne depuis les années 1960. Cet article en analyse les objectifs et les logiques sous-jacentes à travers le prisme du ciblage. La notion de ciblage est ainsi utilisée comme concept analytique pour éclairer les effets territoriaux et sociaux de cette politique. Elle permet également de mettre en perspective les termes du débat actuel autour de la réforme du système. L'analyse menée ici met en relief les dynamiques du ciblage $\mathrm{du}$ point de vue des paysans. Elle ne porte donc pas sur l'évaluation du système de distribution public et les expérimentations de réformes, qui font l'objet d'études spécifiques (Drèze et Khera, 2015; Gulati et Saini, 2015; Joshi et al., 2016; Landy 2011, 2016). Après une brève discussion de la notion de ciblage dans l'agriculture indienne, nous analyserons les effets territoriaux et sociopolitiques pour les paysans. Nous examinerons enfin les enjeux soulevés par l'évolution d'un système qui, selon les décideurs publics, ne serait plus en phase avec les besoins de l'économie indienne.

\section{La notion de ciblage dans l'agriculture indienne: des éléments de catégorisation}

Le ciblage est un processus complexe, recouvrant une grande variété de mécanismes (Lavallée et al., 2009). Pour en comprendre les effets, il est important d'identifier les logiques et les catégorisations qui sont opérées en amont. Maire et al. (1995) distinguent ainsi différentes formes de ciblage que l'on peut utiliser (et combiner) dans les politiques agricoles et alimentaires : le ciblage temporel, géographique, par produit, l'auto-ciblage par utilisation de services, le ciblage au niveau communautaire ou par groupe vulnérable (communauté, âge et sexe) et le ciblage par revenus ou par d'autres critères socioéconomiques. À l'inverse, des mesures non ciblées ou universalistes reviennent à fournir des subventions généralisées. Dans ce sens, les politiques universalistes éviteraient les erreurs d'exclusion de groupes sociaux qui auraient effectivement besoin des mesures de soutien (Swaminathan, 2003). Elles conduiraient aussi à une sorte d'auto-ciblage étant donné que les riches ne sont souvent pas intéressés par les avantages proposés. De fait, cibler les bénéficiaires d'une aide sociale, d'un programme ou d'une politique publique revient à identifier des individus, ménages ou groupes sociaux sur la base de critères socio-économiques, géographiques ou démographiques. Réfléchir aux éléments de catégorisation de la paysannerie indienne permet ainsi de dégager quatre grands axes pour penser les catégories qui peuvent être mobilisées dans le ciblage de la politique agricole du point de vue des paysans.

Premièrement, certaines catégories sociales sont considérées comme désavantagées et bénéficient de mesures de discrimination positive, telles que les prévoit la Constitution de l'Union indienne qui formalise notamment deux catégories : les Tribus répertoriées (Scheduled Tribes) et les Castes répertoriées (Scheduled Castes). À celles-ci viennent s'ajouter les autres catégories défavorisées (Other Backward Classes). L'appartenance à ces catégories permet en principe l'accès à des places réservées dans l'éducation et l'administration publique. Elle va aussi déterminer la participation à certains programmes sociaux en milieu rural. Par exemple le soutien à l'élevage, à travers un programme gouvernemental finançant l'achat de bétail pour les catégories sociales défavorisées, a eu un effet important sur l'augmentation de la production laitière dans les années 1990. Toutefois, si le ciblage direct des catégories défavorisées a été effectué au moyen de programmes sociaux gouvernementaux, dans le domaine de la politique agricole la plupart des instruments utilisés par les pouvoirs publics ne ciblent pas des catégories sociales déterminées.

Deuxièmement, la taille de l'exploitation pourrait constituer un indicateur de départ pertinent pour le ciblage des politiques publiques. La catégorie de la «petite paysannerie », qui comprend les paysans cultivant moins de deux hectares et qui représente $86,6 \%$ des exploitations agricoles en Inde (NSSO, 2014) - ne forme cependant pas un groupe homogène. Ainsi, il est bien différent de posséder un terrain d'un hectare dans une région désertique du Gujarat ou dans une région irriguée du Bengale-occidental. L'agriculture indienne est, lorsque l'on considère la surface cultivée, majoritairement une agriculture pluviale: moins de $40 \%$ des terres cultivées sont irriguées. Ainsi ce critère devrait en principe être rapporté à la région d'origine et la présence ou non d'irrigation, qui déterminent la surface nécessaire pour assurer la viabilité de 
l'exploitation. Cette donnée, couplée à l'analyse de la carte climatique et agro-écologique de l'Inde, peut être utilisée pour une première cartographie de l'agriculture indienne et, partant, des catégories d'exploitations agricoles.

Troisièmement, l'accès aux ressources est un facteur central pour comprendre les catégories utilisées, effectivement ou potentiellement dans les politiques publiques, qu'il s'agisse des ressources en eau, de l'accès à la terre ou du crédit, dont l'insuffisance conditionne la capacité d'investissement des paysans. La spirale de l'endettement est un élément crucial dans la détresse paysanne et le drame des suicides de paysans. Les études réalisées à ce sujet mettent en évidence différents facteurs (Kannan, 2012a, 2012b) : l'endettement, dans un contexte où ils n'ont pas accès au crédit institutionnel, les mauvaises récoltes en lien avec des aléas naturels, les prix élevés pour les intrants et la faible rentabilité de l'agriculture, ainsi que les difficultés liées à la commercialisation. C'est souvent une association de plusieurs facteurs qui met les paysans dans une situation de détresse à laquelle ils ne voient aucune issue.

Quatrièmement, les catégories d'exploitants agricoles tendent à être redéfinies aujourd'hui en fonction du type d'insertion dans le marché. Les travaux d'économistes du développement ont ainsi mis en évidence l'importance du rôle des marchés agricoles, à la fois nationaux et internationaux (Harriss-White, 2008). Il s'agit d'une variable clé pour comprendre les défis et les possibilités de l'agriculture familiale (Bosc et Bélières, 2015 ; Sourisseau et Even, 2015). Ainsi peuton distinguer trois profils-types dans la petite paysannerie indienne (Singh, 2012):

- les paysans qui orientent toute leur production vers la mise sur le marché;

- ceux qui le font dans une moindre mesure, par l'achat des intrants et la vente d'une partie de leur production;

- les paysans qui pratiquent une agriculture de subsistance, autrement dit qui gardent leur production pour l'autoconsommation et achètent un complément sur le marché, devenant ainsi des acheteurs nets de produits alimentaires.

Ces éléments de catégorisation croisée montrent la complexité d'un éventuel ciblage des programmes sur certaines catégories de paysans. Comme le souligne Hazell (2014) «les travaux de recherche distinguent généralement les petites et les grandes fermes, les agriculteurs et les agricultrices, ainsi que les exploitations pauvres et les exploitations riches. Toutefois, il serait plus pertinent, pour mieux cibler les politiques, de reconnaître les différences qui existent au sein de chacun de ces groupes, plutôt qu'entre eux.» D'où la difficulté d'opérer des ciblages qui, comme dans le cas du système d'achat indien, sont censés répondre à un double objectif d'efficacité et d'équité. Or, telle qu'elle a été mise en ouvre depuis la révolution verte, la politique d'achat menée par les pouvoirs publics indiens oscille entre ces deux objectifs, dont les contradictions entre eux deviennent manifestes lors des tentatives de réforme.

\section{Les différentes fonctions du système d'achat public}

En Inde, le marché des grains alimentaires est marqué par l'intervention publique tant dans la régulation des prix que dans l'achat, le stockage, le transport et la distribution de la production. Pour comprendre comment cette intervention a conduit au ciblage de certaines denrées, de certaines régions et de certaines formes de production, il convient de revenir sur la mise en place du système. L'évolution de la politique alimentaire, et plus précisément de la constitution de stocks publics, reflète la transformation d'une intervention de gestion de catastrophes avant l'indépendance de l'Inde lorsque, en 1943, au moment de la famine du Bengale, le Foodgrains Policy Committee, constitué au niveau du gouvernement fédéral, formalise le premier programme d'achat centralisé de grains alimentaires (Saini et Kozicka, 2014) en un système sophistiqué visant à servir de multiples objectifs. Comme le reste de l'Asie du Sud à partir des années 1960, l'Inde mène une politique protectionniste à l'extérieur, et interventionniste à l'intérieur (Dorin et Landy, 2002). Elle cherche à parvenir à un certain degré d'autonomie, en particulier pour les denrées de base, et la constitution de stocks alimentaires permet d'éviter de dépendre de l'aide et des marchés internationaux. Dans cette perspective, la mise en place du système d'achat public s'inscrit d'emblée dans la logique générale de sécurité alimentaire et vise un double objectif: d'une part assurer un environnement de prix rémunérateurs et stables pour les paysans, les encourageant à augmenter la production et favorisant donc la disponibilité des grains alimentaires; d'autre part, il s'agit d'améliorer l'accès économique à l'alimentation pour les plus démunis.

Le système est structuré par trois éléments essentiels liés entre eux, qui représentent en même temps les trois phases de la politique agricole et alimentaire: la définition des prix minimum de soutien (Minimum Support Prices - MSP), l'approvisionnement public par l'agence alimentaire publique (Food Corporation of India - FCI) et la redistribution à travers le système de distribution public. Tout d'abord, les MSP sont annoncés au début de la saison agricole, de façon à servir de garantie aux paysans et à leur permettre d'intégrer cette variable dans leur choix de cultures. Ils correspondent aux prix minimum payés aux paysans pour certaines de leurs productions agricoles, en tout cas lors de leur achat par l'agence alimentaire publique. Les MSP sont calculés sur la base des recommandations faites par la Commission pour les prix et coûts agricoles. Celle-ci considère des facteurs comme le coût de production, les variations dans les prix des intrants, les tendances sur les prix des marchés, l'offre et la demande, ainsi qu'une marge raisonnable pour les paysans. Le MSP fonctionne comme une incitation à la production, des revenus plus élevés encourageant les paysans à investir davantage dans les intrants et les technologies. Le prix final payé aux paysans est le prix d'achat (procurement price), annoncé peu après la récolte. Il correspond au MSP ajusté au besoin (à la hausse uniquement) par le gouvernement central ou par les gouvernements des États. Le système d'achat est ouvert, dans le sens où le gouvernement, par l'intermédiaire de son agence publique, s'engage à acheter toutes les quantités que souhaiteraient vendre les paysans au prix d'approvisionnement. Cet encouragement se cumule avec les subventions non spécifiques à des cultures, qui sont fournies à la fois par l'État central et les États fédérés, comme les subventions aux engrais chimiques et à l'électricité utilisée pour l'irrigation; celle-ci est parfois même gratuite dans certains États (Chatterjee, 2015).

Le second moment correspond à l'achat de la production par l'agence alimentaire publique du gouvernement central 
qui, avec les agences des États, a établi des centres pour l'achat des grains alimentaires. Les stocks ainsi constitués servent à assurer la disponibilité des ressources (rôle de stocks-tampons) et à alimenter le système de distribution public (Public Distribution System - PDS). L'objectif de sécurité alimentaire sur le plan productif se double ainsi d'un volet redistributif, autrement dit d'une intervention publique pour la sécurité alimentaire des consommateurs. La redistribution, troisième phase de la politique, est opérée par les agences gouvernementales dans les 485000 magasins à prix subventionnés (Jha et al., 2010). Aujourd'hui, on estime que le programme couvre 65 millions de familles. L'un des critères essentiels pour le ciblage est celui de la limite de pauvreté : les ménages sous la limite de pauvreté (Below the Poverty Line - BPL) sont distingués de ceux qui sont audessus de la limite de pauvreté (Above the Poverty Line APL). Cependant, le ciblage des consommateurs, variable selon les Etats indiens, ne s'opère pas sans difficultés (Jha et Acharya, 2016; Kishore et Chakrabarti, 2015; Jha et al., 2013). L'élargissement des ayant-droits, avec la mise en œuvre d'un nouveau principe entériné par une loi adoptée en 2013, le Food Security Act, se met progressivement en place. Le système de distribution publique devient ainsi partie intégrante d'un dispositif de protection sociale (Mander, 2015).

Globalement, les opérations d'achat public et les volumes achetés sont influencés par cinq facteurs (Saini et Kozicka, 2014) : les niveaux de production, les incitations de prix (les MSP comparés aux prix du marché), les engagements publics pour alimenter le système de distribution, les restrictions sur les stocks privés de grain et la politique en matière d'import-export du gouvernement fédéral. Cette intervention marquée du «Centre», autrement dit du gouvernement de New Delhi, dans un contexte national où l'agriculture relève des États fédérés, engendre aussi des jeux d'échelle et de pouvoir complexes entre les États et le Centre (Dorin et Landy, 2009). Au départ, les deux objectifs de cette politique étaient de stabiliser l'offre et les prix des grains alimentaires, à la fois dans le temps, à travers des politiques de stockage, et à travers les régions, en achetant des grains aux régions excédentaires pour les acheminer dans les zones déficitaires. La constitution de stocks était utilisée en même temps pour s'attaquer au problème de l'instabilité et de la volatilité des prix sur le marché des grains alimentaires, pour favoriser la sécurité alimentaire et encourager la production.

Le ciblage du volet productif intervient à différents niveaux : si l'approvisionnement public n'est pas orienté vers des catégories déterminées de paysans, il a néanmoins des implications spécifiques pour certaines catégories d'agriculteurs : ceux qui produisent les denrées achetées par l'agence gouvernementale et qui ont les prix d'achat relatifs les plus élevés (souvent qualifiés de "prix rémunérateurs »). En effet, si la Commission pour les prix et coûts agricoles recommande des prix minima de soutien au niveau national pour 24 denrées et productions agricoles, le système de soutien aux prix fonctionne effectivement essentiellement pour le blé et le riz, qui sont concernés par l'achat par l'agence alimentaire publique. De fait, il conduit à une concentration des ressources et donc un ciblage indirect à la fois sur certaines régions et sur certaines catégories de producteurs.

\section{Les implications du système: un effet de ciblage}

L'analyse spatiale du « système alimentaire » de l'Inde met en évidence des objectifs alimentaires, sociaux et productivistes tout en répondant à une «visée éminemment géographique » (Landy, 2009). En alimentant la circulation des grains à travers le territoire indien, l'État met en relation les territoires et les espaces. Cependant, l'intervention massive sur le marché des grains ne se produit pas sur tout le pays de manière homogène, bien que le système d'achat «ouvert» (openended) soit applicable au pays dans son ensemble. Le système opère principalement dans quelques États céréaliers (Pendjab, Haryana, Punjab, Uttar Pradesh et Andhra Pradesh). Les prix d'achat (procurement prices) y deviennent effectivement des prix de soutien, en dessous desquels les prix du marché ne tombent pas (Jha et al., 2010). Alors qu'en principe le MSP existe pour la plupart des cultures, son impact réel reste donc globalement limité à certaines régions agricoles - au demeurant celles qui étaient déjà historiquement associées à la Révolution verte et donc relativement plus prospères depuis plusieurs décennies. L'achat public à un prix minimum de soutien porte sur le blé, le riz et la canne à sucre, cette dernière faisant l'objet d'un système équivalent dans certaines régions. La production de blé est concentrée dans quelques États clés comme le Pendjab, Haryana, Madhya Pradesh et Uttar Pradesh, qui sont à l'origine de près de $90 \%$ de la production du pays (Jha et al., 2010). Quant au riz, bien qu'il s'agisse d'une culture diffusée dans toute l'Inde, six États - Andhra Pradesh, Bihar, Pendjab, Tamil Nadu, Uttar Pradesh et Bengale-occidental - produisent $75 \%$ de la récolte nationale (Jha et al., 2010). Notons cependant que dans le système d'achat public la place relative de ces États peut varier; ainsi le Haryana est un grand producteur mais aussi un grand consommateur de blé et donc son rôle dans le système d'achat public pour cette denrée est moindre que ne pourraient le laisser penser les données de sa production.

Les déséquilibres régionaux sont renforcés par le système d'achat public, qui représente un encouragement à la production pour ces cultures et renforce le poids des régions produisant traditionnellement un surplus de ces céréales. Si les déséquilibres sont manifestes dans les volumes de production achetés par l'agence publique, ils se déclinent aussi à un autre niveau, celui des prix minima de soutien. En réalité, les prix minima de soutien annoncés par les agences publiques sont basés sur une série de facteurs non économiques, comme l'influence politique des gouvernements fédérés et des lobbies paysans. Les MSP qui, rappelons-le, peuvent être ajustés à la hausse par les États fédérés, deviennent dès lors un enjeu politique. Les augmentations sont continues et elles sont très marquées dans certains États agricoles, comme le Pendjab, le Haryana ou l'Uttar Pradesh, autrement dit ceux qui fournissent en grande partie le système d'approvisionnement. Les demandes de prix «plus rémunérateurs » sont une constante des mobilisations des mouvements paysans qui, depuis une trentaine d'années, organisent les paysans producteurs de surplus commercialisables dans les régions agricoles les plus prospères (Lutringer, 2012, 2015). Ainsi, lors d'une manifestation du Karnataka Rajya Raitha Sangha (KRSS) en janvier 2015 organisée devant le bureau agricole régional de Bangalore, la capitale de l'État du Karnataka, le mouvement 
paysan demande que le gouvernement fixe des «prix scientifiques » pour la production agricole (Sivandandan, 2015). Les prix fixés par le gouvernement sont jugés trop bas et même inférieurs au coût des intrants. Si la question du prix «juste», « rémunérateur» ou «scientifique» préoccupe les agriculteurs et leurs représentants, elle fait également débat parmi les économistes et analystes politiques (NITI Aayog, 2015). Cela révèle la multiplication des fonctions attribuées aux MSP, qui sont compris comme un instrument visant à garantir un revenu aux paysans. En témoignent aussi les propositions de réformes émanant de la National Commission on Farmers ; cette commission, dite Swaminathan car présidée par le célèbre agronome, a recommandé d'augmenter les prix minima de soutien de $50 \%$ par rapport aux coûts de production moyens (Singh Ghuman, 2015). Mais encore une fois, l'effectivité des MSP sur le terrain étant liée à la politique d'achat public, elle-même concentrée dans certaines régions agricoles, il s'agit d'un instrument, de fait, ciblé, qui concentre spatialement les ressources allouées à une politique donnée. Enfin, notons l'effet de ciblage parallèle sur certaines catégories de producteurs: les exploitations tournées vers l'autosubsistance jouant un rôle tout à fait mineur dans la production d'excédents commercialisables et donc dans l'approvisionnement public, ce sont les paysans producteurs de surplus qui, de fait, représentent les principaux bénéficiaires du système d'achat à prix garanti.

Les déséquilibres, à la fois en termes de volumes de production et de prix, engendrés par le système ont justifié des tentatives de réforme. D'une part, les stocks de riz et de blé sont largement supérieurs aux objectifs d'achat de l'agence publique. D'autre part, alors que la production indienne de légumineuses et d'oléagineux ne suffit pas à répondre à la demande interne et que les prix payés sur le marché sont sujets à une inflation récurrente, les prix reçus par les paysans sur le marché interne tombent paradoxalement souvent en dessous du MSP. On constate que le seul instrument du MSP ne suffit pas : dans un contexte où les aléas de la communication et des transports inter-régionaux entravent la circulation des marchandises agricoles et le jeu des prix, le manque d'intégration des marchés agricoles régionaux pèse aussi sur la question des prix (Landy, 2011, 2016).

\section{Vers un ciblage plus explicite?}

Les réformes et rééquilibrages esquissés par différents gouvernements successifs depuis la fin des années 1980 sont liés à différents constats, du point de vue économique, financier et nutritionnel. En effet, la croissance dans la production domestique a eu un impact limité sur la disponibilité et la consommation alimentaire (Ganesh-Kumar et al., 2010; Taravella et Barbier, 2002). Sur le plan productif, l'engagement de l'État dans la promotion d'autres cultures, notamment les oléagineux à travers la «révolution jaune» initiée dans les années 1980 (Dorin et Landy, 2002), les fruits, légumes et produits horticoles ainsi que les légumineuses, vise à accompagner ou favoriser la diversification de la production qui est en cours dans les campagnes indiennes (Etienne, 2014). Par ailleurs, la décentralisation dans les opérations d'achat et de distribution par l'agence publique alimentaire a pour objectif de réduire les coûts de fonctionnement et les pertes lors de l'approvisionnement et du transport. Il s'agit par-là d'améliorer l'efficacité du système de distribution publique (Sharma et Alagh, 2013). Comme le montre la minutieuse analyse effectuée par Harriss-White (2008) au Bengaleoccidental, le premier État à remplacer la FCI par sa propre agence en 1997, les facteurs politiques internes aux États et les formes de régulation sociale traditionnelles ont un impact prégnant sur le fonctionnement des marchés agricoles régionaux. Dans ce contexte, l'éventuel objectif d'équité sociale associé à l'intervention publique est difficile à atteindre. Au demeurant, les instruments de prix dont disposent les États sont sujets à une pression des lobbies et mouvements paysans déjà évoqués plus haut.

Au niveau fédéral, l'argument de la charge financière du système d'achat public est récurrent chez les partisans d'une réforme. Le coût total, qui correspond à ce que l'on entend par subventions alimentaires (food subsidy), est composé de trois éléments: le prix payé aux paysans, les frais liés à l'approvisionnement et le coût de distribution. Le gouvernement estime que les coûts liés à l'achat, au stockage et à la distribution de grains alimentaires sont de 40-50\% supérieurs au prix d'achat proprement dit (procurement price) (Government of India, 2015). De l'autre côté, le prix de vente dans les magasins subventionnés (issue price) est resté inchangé depuis 2002 (Government of India, 2015). Cet argument d'une charge financière excessive, scandé depuis une vingtaine d'années par certains dirigeants au pouvoir et par certains centres de recherche indiens et internationaux, a contribué à justifier, en août 2014, la création d'une commission chargée d'évaluer la politique ouverte d'achat public. Par ailleurs, l'accent sur les régions de la Révolution verte a favorisé des cultures marquées par une utilisation intensive de l'eau et des engrais. Ce mode de production est de plus en plus sujet à discussion (Radja et al., 2016), compte-tenu de son impact sur l'environnement (pollution des sols et des eaux souterraines par les engrais chimiques, baisse de la nappe phréatique) et, en même temps, de la stagnation des rendements. L'exemple du Pendjab est révélateur : région de Révolution verte par excellence, elle est à la recherche d'un renouveau de son modèle de développement. On y observe que la croissance de l'agriculture vient à présent de plus en plus de facteurs non liés aux prix (Singh et Singh, 2016).

La commission instituée par le gouvernement de New Delhi (High Level Committee on restructuring of Food Corporation of India) présidée par Shanta Kumar, a rendu un rapport suggérant de restructurer l'agence d'approvisionnement public afin d'améliorer son efficacité opérationnelle et sa gestion financière. Des éléments importants de ces recommandations ont ensuite été repris dans les rapports économiques annuels (Economic Surveys) du gouvernement indien. Des expressions fortes sont utilisées comme «donner un nouveau visage à la FCI » ou « créer de la concurrence dans chaque étape du marché alimentaire », révélant un changement de paradigme. La FCI change de nature, son rôle est présenté non plus tellement en lien avec la sécurité alimentaire mais avec le bon fonctionnement des marchés agricoles. Concrètement, la Commission recommande également de limiter l'approvisionnement venant d'États qui décrètent un bonus supérieur au MSP et qui fournissent des quantités supérieures à celles requises par le système de distribution public régional. En même temps, d'autres voix plaident pour étendre 
l'approvisionnement public à d'autres cultures, dans l'idée qu'une intervention forte sur les prix alimentaires est importante pour contenir l'inflation. Ainsi Raghuram Rajan, alors gouverneur de la Banque centrale indienne, encourage à constituer des stocks publics de légumineuses et d'oléagineux (Ninan, 2015). Dans son rapport économique annuel de 2016, le gouvernement indique lui aussi que la politique des MSP devrait être réorientée vers les légumineuses afin d'augmenter leur production.

Il semble donc que l'achat public et les MSP soient toujours centraux dans le débat sur l'intervention publique en matière agricole, et ce même si certaines propositions de réforme remettraient théoriquement en cause les instruments utilisés jusqu'à présent: en particulier, si les transferts financiers étaient adoptés et se généralisaient, les subventions aux plus pauvres remplaceraient les stocks à distribuer, ce qui viendrait sérieusement questionner la pertinence du système d'achat auprès des paysans. Toutefois, la perspective d'un changement de cette nature est encore éloignée, les réformes actuelles visant plutôt à calculer le MSP en fonction de nouveaux paramètres: le rapport économique annuel du gouvernement encourage à donner la priorité aux cultures qui requièrent moins d'eau, comme les légumineuses et les oléagineux, et envisage pour celles-ci un système d'achat public. Selon cette vision, le MSP devrait augmenter en fonction des externalités positives ou des bénéfices sociaux liés à ces productions. À l'inverse, dans le cas de la culture de blé, du riz ou de la canne à sucre, le MSP tiendrait compte des externalités négatives dérivant de l'utilisation d'engrais chimiques et de la diminution du niveau des nappes phréatiques.

Les propositions de réforme visent aussi à cibler les régions récipiendaires, cette fois-ci de manière plus explicite et dans une logique inverse par rapport à ce qui a été pratiqué jusqu'à présent. Le rapport du gouvernement fait référence aux régions d'Inde orientale, caractérisées par une agriculture moins intensive que les régions de Révolution verte et où le climat semi-aride et les faibles niveaux d'irrigation rendent sujettes aux aléas de la mousson. Or l'inclusion des millets et des légumineuses, cultures qui sont adaptées à leurs conditions agro-climatiques, dans le système d'achat public, permettrait de répondre à un double objectif: augmenter les revenus des petits paysans et s'attaquer à la malnutrition rurale en augmentant la diversité alimentaire (Government of India, 2016a, b).

Le ciblage proposé correspond à un rééquilibrage : il s'agit d'accorder plus de poids aux petits États qui ne sont pas les fournisseurs traditionnels du système d'approvisionnement public. Ainsi l'agence gouvernementale centrale devrait-elle céder toutes les opérations d'approvisionnement de blé et de riz aux États qui ont acquis une expérience suffisante et qui ont des infrastructures adéquates. Elle n'accepterait les surplus, après déduction des besoins des États au titre du programme de redistribution alimentaire, que de ces gouvernements-là. Enfin, le gouvernement exhorte la FCI à n'aider que «les États dont les paysans sont contraints de vendre leurs productions à des prix inférieurs aux MSP et qui sont dominés par des petites exploitations agricoles»(Government of India, 2016a). Ce faisant, il introduit pour la première fois la notion d'un ciblage explicite, à la fois sur des régions agricoles traditionnellement défavorisées ou délaissées par l'intervention publique, et sur des catégories de paysans. Mais cela pose la question de ce que recouvre la catégorie des «petites exploitations agricoles», une catégorie qui, on l'a vu, est loin d'être homogène. Il semblerait au contraire de plus en plus pertinent de croiser les catégorisations sociales et géographiques: pour les petits exploitants agricoles, les opportunités économiques sont généralement plus importantes dans les régions agricoles dynamiques. En fait, on observe « un ensemble de situations plus variées et plus polarisées, qui doit être pris en compte lors du ciblage des investissements agricoles » (Hazell, 2014). Ce constat appelle-t-il à remettre en question la pertinence de la catégorisation en «petites exploitations agricoles»? En tout cas, cet état de fait, qui n'est pas propre à l'Inde mais qui est particulièrement bien illustré par les données récoltées dans le sous-continent, incite à promouvoir des approches plus fines pour cartographier et catégoriser les régions agricoles, à la fois en termes d'agro-écologie, d'accès au marché et de bénéficiaires, actuels ou potentiels, d'interventions publiques. Ceci à un moment où, pour la première fois, le débat sur le système d'achat public indien porte sur un ciblage explicite, à la fois en termes de régions agricoles et de catégories de paysans.

\section{Conclusion}

Au long d'un demi-siècle d'existence, le système indien d'achat public et de soutien aux prix agricoles a permis de répondre à un certain nombre de défis, en stimulant la production céréalière du pays et en renforçant une dynamique de modernisation agricole, en particulier dans les régions de Révolution verte. Du point de vue des paysans, le bilan de cette politique est plus mitigé lorsque l'on effectue une analyse à la fois spatiale et sociale : les disparités régionales, en termes de performances agricoles ou de structures agraires, persistent et se sont même renforcées. Concrètement, en Inde, l'intervention de l'État dans le secteur agricole est largement construite autour de ce système d'achat public d'une partie des récoltes céréalières, combiné à une politique de prix régulés (théoriquement en tout cas) pour un certain nombre de produits. Si la plupart des mesures liées à ce système correspondent à des mesures ouvertes, elles ont néanmoins conduit à un ciblage à la fois régional, par produit et de certaines catégories de producteurs. Alors qu'elles ont concentré une grande partie des ressources budgétaires dévolues à l'agriculture, au même titre que les subventions à l'électricité et aux intrants, elles ont été assorties de mesures de rééquilibrage. Aujourd'hui le débat sur la réforme de ce système, et surtout la position officielle du gouvernement de New Delhi, sont formulés en termes de ciblage explicite. Il s'agirait par là de rééquilibrer l'allocation des ressources et de donner un rôle plus important à d'autres cultures et à d'autres régions, jusque-là situées à la périphérie du développement agricole indien. Cette exhortation n'est pas nouvelle, de nombreux chercheurs et praticiens l'ayant relayée depuis longtemps. Pour ce faire, ne faudrait-il pas, à l'heure où beaucoup réfléchissent en termes de « smart policies » (Gautam, 2016), cibler plutôt, comme le propose Landy (2013), des modes de production agricole durables tels que l'agriculture biologique, souvent pratiquée dans les régions agraires hors des zones de Révolution verte et par des petits paysans qui n'ont pas les moyens d'investir en intrants coûteux? Toutefois, si plusieurs objectifs sont mentionnés à la faveur de la réforme souhaitée par 
les pouvoirs publics (diminution des coûts, encouragement d'une production plus respectueuse de l'environnement), il semble bien que l'argument central du ciblage envisagé par le gouvernement actuel soit moins celui d'une catégorisation en faveur d'une plus grande justice sociale que la recherche d'une meilleure efficacité du système productif agricole dans son ensemble. En tout cas, le passage d'une notion de ciblage indirect ou implicite, à un ciblage explicite a le mérite de permettre de distinguer plus clairement les différents objectifs de l'intervention publique.

Remerciements. L'auteure souhaite remercier les deux lecteurs anonymes pour leurs commentaires importants et constructifs, qui ont été intégrés dans la présente version de l'article.

\section{Références}

Bosc J-M., Bélières J-F. 2015. Transformations agricoles : un point de vue renouvelé par une mise en perspective d'approches macro et microéconomiques. Cahiers Agricultures 24(4): 206-214. DOI: https://doi.org/10.1684/agr.2015.0762

Chatterjee S. 2015. Powering' agriculture: Andhra Pradesh's free electricity story. Economic and Political Weekly 50(44): 18-21.

Dorin B, Aubron C. 2016. Croissance et revenu du travail agricole en Inde : une économie politique de la divergence (1950-2014). Économie Rurale 352: 41-65.

Dorin B, Landy F. 2002. Agriculture et alimentation de l'Inde: Les vertes années (1947-2001). Paris: INRA éditions.

Dorin B, Landy F. 2009. Agriculture and food in India. A half-century review, from independence to globalization. New Delhi: Quae, $\mathrm{CSH}$, Manohar.

Drèze J, Khera R. 2015. Understanding Leakages in the public distribution system. Economic and Political Weekly 50(7) : 39-42.

Etienne G. 2014. Indian villages. Achievements and alarm bells, 19522012. Genève: Graduate Institute Publications, coll. «eLivres de 1'Institut».

Ganesh-Kumar A, Roy D, Gulati A. 2010. Introduction. In: GaneshKumar A, Roy D, Gulati A, eds. Liberalizing Foodgrains Markets. Experiences, Impact and Lessons from South Asia. New Delhi: Oxford University Press.

Gautam M. 2016. Making Indian agriculture more resilient: Some policy priorities. Economic and Political Weekly 51(8): 24-27.

Government of India. 2015. Economic Survey 2014-2015. Available from: http://indiabudget.nic.in/vol1_survey.asp.

Government of India 2016a. Economic Survey 2015-2016. Available from: http://indiabudget.nic.in/vol1_survey.asp.

Government of India 2016b. Agricultural statistics at a glance 2015, Ministry of Agriculture. Available from: http://eands.dacnet.nic.in/ PDF/Pocket-Book2015.pdf.

Gulati A, Saini S. 2015. Leakages from public distribution system (PDS) and the way forward. Working Paper 294, Indian Council for Research on International Economic Relations, New Delhi.

Harriss-White B. 2008. Rural commercial capital: Agricultural markets in West Bengal. New Delhi: Oxford University Press.

Hazell P. 2014. Repenser le rôle des petites exploitations agricoles dans les stratégies de développement, Point de vue $n^{\circ} 2$, FARM.

Jha P, Acharya N. 2016. Public provisioning for social protection and its implications for food security an analysis. Economic and Political Weekly 51(18): 98-106.

Jha S, Srinivasan PV, Ganesh-Kumar A. 2010. Achieving food security in a cost-effective way: Implications of domestic deregulation and liberalized trade in India. In: Ganesh-Kumar
A, Roy D, Gulati A, eds., Liberalizing foodgrains markets. Experiences, impact and lessons from South Asia. New Delhi: Oxford University Press.

Jha R, Gaiha R, Pandey MK, Kaicker N. 2013. Food subsidy, income transfer and the poor: A comparative analysis of the public distribution system in India's states. Journal of Policy Modeling 35: 887-908.

Joshi A, Sinha D, Patnaik B. 2016. Credibility and portability? Lessons from CORE PDS reforms in Chhattisgarh. Economic and Political Weekly 51(37): 51-59.

Kannan E. 2012a. Trends in India's agricultural growth and its determinants. Asian Journal of Agriculture and Development 8(2): 79-99.

Kannan E. 2012b. Wither farm profitability? A cause of agrarian distress in India. In: Proceedings of National Seminar on Agrarian Distress in India: The Way Out. National Institute of Rural Development, Hyderabad (India), 5-7 Novembre 2012b, pp. 517 534.

Kishore A, Chakrabarti S. 2015. Is more inclusive more effective? The 'New Style' public distribution system in India. Food Policy 55: $117-130$.

Landy F. 2006. Un milliard à nourrir : grain, territoire et politique en Inde. Paris : Belin.

Landy F. 2011. Représentation du territoire national et circulation des grains : le système de distribution publique indien. Annales de géographie 677: 26-49.

Landy F. 2013. From trickle down to leapfrog. How to go beyond the green revolution? Economic and Political Weekly 48(24): 42-49.

Landy F. 2016. Rescaling the public distribution system in India: Mapping the uneven transition from spatialization to territorialization. Environment and Planning C: Government and Policy 35 (1): 113-129.

Lavallée E, Olivier A, Pasquier-Doumer L, Robillar AS. 2009. Le ciblage des politiques de lutte contre la pauvreté : quel bilan des expériences dans les pays en développement? Développement, Institutions et Analyses de Long Terme (DIAL). Paris.

Lutringer C. 2012. Gouvernance de l'agriculture et mouvements paysans en Inde. Paris et Genève: Karthala et The Graduate Institute Publications.

Lutringer C. 2015. Uneven development and peasant mobilisations: Perspectives from Chhattisgarh and Uttar Pradesh. In: Basile E, Harriss-White B, Lutringer C, eds. Mapping India's Capitalism: Old and New Regions. Basingstoke: Palgrave Mcmillan.

Maire B, Delpeuch F, Padilla M, Le Bihan G. 1995. Le ciblage dans les politiques et programmes nutritionnels. In: Padilla M, Delpeuch F, Le Bihan G, Maire B, eds. Les politiques alimentaires en Afrique $d u$ Nord : d'une assistance généralisée aux interventions ciblées. Paris: Karthala, pp. 35-57.

Mander H. 2015. L'approvisionnement alimentaire public en tant que protection sociale. Débattre de la loi sur la sécurité alimentaire nationale de l'Inde. Rome: FAO.

Ninan O. 2015. Rajan moots minimum support price across commodities. The Hindu, 2 June.

NITI Aayog, Government of India. 2015. Raising Agricultural Productivity and Making Farming Remunerative for Farmers, Occasional Paper.

NSSO. 2014. Key indicators of situation assessment of agricultural households. 70th Round, January-December 2013, National Sample Survey Office, Ministry of Statistics and Programme Implementation.

Pouch T, Kheraief N. 2016. Le commerce extérieur agroalimentaire de l'Inde. Économie Rurale 352: 67-80. 
Radja K, Schembri P, Bazin D. 2016. Quels enjeux de soutenabilité pour l'agriculture indienne? Économie Rurale 352: 23-39.

Saini S, Kozicka M. 2014. Evolution and critique of buffer stocking policy of India. Working Paper 283, Indian Council for Research on International Economic Relations (ICRIER).

Sharma VP, Alagh M. 2013. Food subsidy in India: Its components, trends, causes and reforms for public policy. Indian Journal of Agricultural Economics 68(2): 195-221.

Singh Ghuman R. 2015. Swaminathan MSP Solution to Agrarian Crisis and Farmers' Distress? Economic and Political Weekly 50 (33): 20-23.

Singh S. 2012. New markets for smallholders in India, exclusion, policy and mechanisms. Economic and Political Weekly 47(52): 95-105.
Singh L, Singh N, eds. 2016. Economic transformation of a developing economy: The experience of Punjab, India. Singapour: Springer.

Sivandandan TV, 2015. Farmers block roads, protest against government's policies. The Hindu, 3 January.

Sourisseau J-M., Even M-A. 2015. Transformations agricoles et agricultures familiales : quelques défis mis en lumière durant l'année internationale de l'agriculture familiale. Cahiers Agricultures 24(4): 201-203. DOI: https://doi.org/10.1684/agr.2015.0764.

Swaminathan M. 2003. Strategies towards Food Security. Social Scientist 31(9/10): 58-94.

Taravella R, Barbier B. 2002. Les spécificités de la consommation alimentaire en Inde. Étude bibliographique pour Pluriagri, Rapport d'étape 71.

Citation de l'article : Lutringer C. 2017. Le ciblage des politiques agricoles en Inde: les implications pour les paysans du système d'approvisionnement public. Cah. Agric. 26: 45008. 